November 1995

\title{
THE MAGNUS FORCE ON SKYRMIONS IN FERROMAGNETS AND QUANTUM HALL SYSTEMS
}

\author{
Michael Stone \\ Department of Physics \\ University of Illinois at Urbana Champaign \\ 1110 W. Green St. \\ Urbana, IL 61801 \\ USA
}

\begin{abstract}
The topological solitons, or "skyrmions", in a planar ferromagnet experience a Magnus force proportional to the product of their velocity and the surrounding magnetization. It has been suggested that the charged quasiparticles near filling factor $\nu=1$ in the GaAs quantum Hall effect are skyrmions. If so, we might expect this spin-induced Magnus force to act on the quasiparticles in addition to the Lorentz force they experience because of their charge. We show that this is not the case, and that the Magnus and Lorentz forces are merely different descriptions of the same physical effect.
\end{abstract}




\section{Introduction}

Sondhi et al. have argued [1] that the lowest energy charged quasiparticles near filling fraction $\nu=1$ in the GaAs quantum Hall effect are topological solitons, or "skyrmions". The $\nu=1$ ground-state is a filled spin-polarized Landau level. For non-interacting electrons the elementary excitations consist of the addition of a single electron with reversed spin, or the removal of a single electron from the fully polarized Landau level. The change in spin from the addition or removal of an electron is in each case $\Delta S_{z}=-\frac{1}{2}$. Skyrmions are different. They consist of an extended region where the spin direction gradually twists. This slowly varying spin texture serves to bind or repel a unit charge, so the skyrmions still have charge $q= \pm e$, but their total spin is much larger than $\frac{1}{2}$. They are not perturbatively related to the single-particle elementary excitations. Skyrmions will be energetically favored over the $S_{z}=\frac{1}{2}$ quasiparticles whenever sharply localized charge fluctuations require more energy than overturning a number of spins -i.e. when the gyromagnetic ratio $g$ is small [1].

The skyrmion picture has received strong support from recent measurements by Barrett et al. [2]. These authors see a precipitous fall in the spin polarization of the electron gas on both sides of $\nu=1$. This clearly indicates that many spins are being overturned by the addition or removal of a single electron.

By looking for motional narrowing effects it is possible that the NMR methods used in [2] could probe the mobility of the quasiparticles. Before attempting to calculate the mobility by taking into account dissipative effects and quantum diffusion it is however necessary to have a thorough understanding of the quasi-classical forces acting on a moving skyrmion. Now the skyrmion configurations in a conventional ferromagnet experience a "Magnus" force proportional to the product of their velocity and the local magnetization. This force prevents the skyrmion from moving with respect to the spin background. Because the skyrmion quasiparticles in the quantum Hall effect are electrically charged we would expect them to experience a Lorentz force in addition to the Magnus force. This Lorentz force is of the same magnitude as the Magnus force and one might hope that the two forces would cancel, allowing the skyrmions to move freely. Sadly this does not happen. It is easy to see that the two forces are identical in both magnitude and sign. This equality is no coincidence. There is really only one force, and its two apparently distinct origins are merely differing interpretations of a single geometric phase. Only one of the two forces should therefore be taken into account when considering the motion of the skyrmions. The present paper is devoted to a discussion of this.

In section two we review the lagrangian approach to the dynamics of a spin. We then show how the Magnus force appears in a conventional ferromagnet composed of neutral spins obeying the Landau-Lifshitz 
equation. In section three we show how Landau-Lifshitz dynamics arises in a simple model for the Hall effect. We then use a duality transformation to demonstrate that the Lorentz force is merely the Magnus force in disguise. The last section provides a simple physical explanation of why there is only one force.

\section{Ferromagnets}

To establish our notation we start with a brief review of the dynamics of a single spin, whose direction we denote by the unit vector $\mathbf{n}$. The classical action for a spin in a magnetic field $\mathbf{B}$ is a functional of the spin trajectory, or history, $\mathbf{n}(t)$ and is given by $[3,4]$

$$
S=-J \int \dot{\mathbf{n}} \cdot \mathbf{A}(\mathbf{n}) d t+\mu \int \mathbf{B} \cdot \mathbf{n} d t
$$

The second term in this expression is simply $-\int H d t$ where $H=-\mu \mathbf{B} \cdot \mathbf{n}$ is the hamiltonian of for a spin of moment $\mu \mathbf{n}$ in the field $\mathbf{B}$. The first term is more complicated. Here $\mathbf{A}(\mathbf{n})$ denotes the gauge potential of a unit (flux $=4 \pi)$ "magnetic" monopole located at the center of the unit sphere $S^{2}$ on which $\mathbf{n}$ lies. For open trajectories this term depends on the particular gauge chosen for $\mathbf{A}$, but when the motion of the $\mathbf{n}$ vector is required to be periodic, as for example when computing partition functions, we can rewrite (2.1) in a manifestly gauge invariant manner as [5]

$$
S=-J \iint \mathbf{n} \cdot\left(\partial_{\tau} \mathbf{n} \wedge \partial_{t} \mathbf{n}\right) d \tau d t+\mu \oint \mathbf{B} \cdot \mathbf{n} d t
$$

The coordinates $t$ and $0 \leq \tau \leq 1$ parameterize the interior of the region $\Gamma \subset S^{2}$ bounded by the curve $\mathbf{n}(t)$ on which $\tau=1$. We have extended the definition of the function $\mathbf{n}(t)$ to a function $\mathbf{n}(t, \tau): \Gamma \rightarrow S^{2}$ such that $\mathbf{n}(t, 1)=n(t)$. The numerical value of the action $S$ is independent of the choice of extension. Geometrically, the first term in (2.2) is simply the oriented area of the region $\Gamma$.

The classical equation of motion is found by varying $\mathbf{n}(t)$ in (2.2). The variation of the first term is most easily obtained from its geometric interpretation. We evaluate the change in the area of $\Gamma$ due to the variation in its boundary and so find

$$
\delta S=-J \oint(\mathbf{n} \cdot(\delta \mathbf{n} \wedge \dot{\mathbf{n}})) d t+\mu \oint \mathbf{B} \cdot \delta \mathbf{n} d t
$$

In order to respect the constraint on the length of $\mathbf{n}$ we can write $\delta \mathbf{n}=\mathbf{n} \wedge \delta \mathbf{w}$, whence

$$
\delta S=\oint \delta \mathbf{w} \cdot(J \dot{\mathbf{n}}-\mu(\mathbf{n} \wedge \mathbf{B})) d t
$$

The action principle therefore tells us that

$$
J \dot{\mathbf{n}}-\mu(\mathbf{n} \wedge \mathbf{B})=0 .
$$


We see that the spin undergoes the desired Larmor precession about the direction of the $B$ field.

The role of the "monopole" is made clear by acting on (2.5) with $\mathbf{n} \wedge$ to get

$$
J(\dot{\mathbf{n}} \wedge \mathbf{n})+\mu(\mathbf{B}-(\mathbf{B} \cdot \mathbf{n}) \mathbf{n})=0 .
$$

The first term in (2.6) can now be interpreted as the "Lorentz" force on a particle of "charge" $J$ constrained to move on $S^{2}$ in the field of the monopole. The other term, the component of $\mu \mathbf{B}$ tangent to the sphere, is the force attempting to align the spin along the direction of $\mathbf{B}$. The particle is massless so the two forces acting on it must add to zero.

When the system is quantized by placing the classical action in the exponent of a path integral $\mathcal{Z}=$ $\int d[\mathbf{n}] \exp i S$, the ambiguity in the region ("inside" vs, "outside") bounded by $\mathbf{n}(t)$ requires $J$ to take integer or half-integer values, giving rise to the familiar quantization of angular momentum. The quantization of spin by this method is part of the general theory of group representations via the method of co-adjoint orbits $[6]$.

We can immediately extend these ideas to a continuum model of a ferromagnet with $\rho$ spins per unit area, each of magnitude $J$. The only modifications required are to make $\mathbf{n}$ a function of position as well as time, and to replace the external $\mathbf{B}$ field by a spin-stiffness term. We therefore take

$$
S=-J \rho \int \dot{\mathbf{n}} \cdot \mathbf{A}(\mathbf{n}) d t d^{2} x-\frac{1}{2} K \int(\nabla \mathbf{n})^{2} d t d^{2} x
$$

or equivalently, for periodic histories,

$$
S=-J \rho \int\left(\mathbf{n} \cdot\left(\partial_{\tau} \mathbf{n} \wedge \partial_{t} \mathbf{n}\right)\right) d \tau d t d^{2} x-\frac{1}{2} K \int(\nabla \mathbf{n})^{2} d t d^{2} x
$$

The corresponding equation of motion,

$$
J \rho \dot{\mathbf{n}}-K \mathbf{n} \wedge \nabla^{2} \mathbf{n}=0
$$

is the Landau-Lifshitz equation [7] describing the precession of each $\mathbf{n}$ in the field of its neighbors.

The equation $\nabla^{2} \mathbf{n}=0$ has topological soliton solutions, or "skyrmions" where the mapping $\mathbf{n}(\mathbf{r}): \mathbf{R}^{2} \rightarrow$ $S^{2}$ covers the sphere once as the point $\mathbf{r}=\left(x^{1}, x^{2}\right)$ covers the plane. Using spherical polar coordinates $\theta, \phi$ to parameterize $S^{2}$ these solutions can be written

$$
e^{i \phi} \cot \theta / 2=\frac{a}{z}
$$


where $z=x^{1}+i x^{2}$. For anti-skyrmions we replace $z$ by its conjugate. The spins in (2.10) point up at the origin and gradually tilt down as one moves outwards. They point straight down at infinity.

It is fairly easy to show [8] that a multi-skyrmion solution to $\nabla^{2} \mathbf{n}=0$ is given by

$$
e^{i \phi} \cot \theta / 2=f(z)
$$

where $f(z)$ is any rational function of $z$. The number of skyrmions - i.e. the degree or winding number of the map $\mathbf{R}^{2} \rightarrow S^{2}$ - is given by the number of poles (equivalently by the number of zeros) of $f(z)$. For the simple stiffness term in (2.6) the strain energy of these multi-skyrmion solutions is independent of the parameters in the rational function. In particular single skyrmions of different scale $a$ are all degenerate in energy. The skyrmions in the quantum Hall effect have a definite size determined by a competition between coulomb repulsion and the Zeeman energy [1]. They are more compact than the solutions given by (2.10).

Now we investigate the mobility of the skyrmions. Let us denote the spin configuration of a skyrmion centered at the origin by $\mathbf{n}_{0}(\mathbf{r})$. We introduce a collective coordinate $\mathbf{R}=\left(R^{1}, R^{2}\right)$ so that the spin field of a moving skyrmion can be written, at least as a first approximation, as $\mathbf{n}(\mathbf{r}, t)=\mathbf{n}_{0}(\mathbf{r}-\mathbf{R}(t))$. We insert this ansatz into (2.8) and see what action it costs to move the skyrmion round a closed path in the plane.

We therefore wish to evaluate

$$
S=-\rho J \int \dot{\mathbf{n}}(\mathbf{r}, t) \cdot \mathbf{A}(\mathbf{n}(\mathbf{r}, t)) d t d^{2} x
$$

with $\mathbf{n}(\mathbf{r}, t)=\mathbf{n}_{0}(\mathbf{r}-\mathbf{R}(t))$. It is actually more convenient to consider the variation of (2.12) under a small perturbation in the path $\mathbf{R}(t)$. We then use

$$
\delta S=-\rho J \int \mathbf{n} \cdot(\delta \mathbf{n} \wedge \dot{\mathbf{n}}) d t d^{2} x
$$

with

$$
\dot{\mathbf{n}}=-\frac{\partial}{\partial x^{i}} \mathbf{n}_{0}(\mathbf{r}-\mathbf{R}) \dot{R}^{i},
$$

and

$$
\delta \mathbf{n}=-\frac{\partial}{\partial x^{i}} \mathbf{n}_{0}(\mathbf{r}-\mathbf{R}) \delta R^{i} .
$$

Thus

$$
\begin{aligned}
\delta S & =-\rho J \int \mathbf{n}_{0}(\mathbf{r}-\mathbf{R}) \cdot\left(\partial_{i} \mathbf{n}_{0}(\mathbf{r}-\mathbf{R}) \wedge \partial_{j} \mathbf{n}_{0}(\mathbf{r}-\mathbf{R})\right) \delta R^{i} \dot{R}^{j} d t d^{2} x \\
& =-\rho J \int \delta R^{i} \dot{R}^{j}\left\{\int \mathbf{n}_{0}(\mathbf{r}-\mathbf{R}) \cdot\left(\partial_{i} \mathbf{n}_{0}(\mathbf{r}-\mathbf{R}) \wedge \partial_{j} \mathbf{n}_{0}(\mathbf{r}-\mathbf{R})\right) d^{2} x\right\} d t .
\end{aligned}
$$


The factor in braces in (2.16) is independent of $R$ and is equal to $4 \pi \mathcal{N} \epsilon_{i j}$ where $\mathcal{N}$ is the degree of the map $\mathbf{n}: \mathbf{R}^{2} \rightarrow S^{2}$ i.e. the skyrmion number. We therefore have that

$$
\delta S=-4 \pi \mathcal{N} \rho J \oint\left(\delta R^{1} \dot{R}^{2}-\delta R^{2} \dot{R}^{1}\right) d t
$$

This is the variation of

$$
S=-2 \pi \mathcal{N} \rho J \oint\left(R^{1} \dot{R}^{2}-R^{2} \dot{R}^{1}\right) d t
$$

When used in the path integral $\mathcal{Z}=\int d[\mathbf{n}] \exp i S,(2.18)$ means that the skyrmion accumulates a phase of $2 \pi$ for every spin- $\frac{1}{2}$ it encircles. The physical consequence of this phase may be recognized by noting that the right hand side of (2.18) is the same term that occurs in the action for a particle of charge $\mathcal{N}$ moving in a uniform magnetic field of strength $2 \pi \rho J$. Like the charged particle therefore, the skyrmion must experience a transverse "Lorentz" or "Magnus" force proportional to its velocity. We will use the latter designation because the force is proportional to the surrounding spin density.

The Magnus force pins the skyrmion in place just as an electron in the lowest Landau level is pinned in place by the quenching of its kinetic energy. The addition to the lagrangian of an inertial term proportional to $|\dot{\mathbf{n}}|^{2}$ would induce a mass for the skyrmion and permit it to make cyclotron orbits, but in the absence of scattering its wandering would still be strongly restricted. A gradient in an additional Zeeman term will however lead to the skyrmion drifting along the skyrmion's Zeeman-energy contours at such a speed that the Magnus force balances the Zeeman-energy gradient.

At first sight it is surprising that we accumulate a phase proportional to the area encircled by the skyrmion. The phase comes from a term involving the space integral of $\dot{\mathbf{n}}$, and far from the skyrmion the spins do not move. Naively this would lead us to expect a phase proportional to at most the length of the skyrmion trajectory, and not to the area it encloses. The incipient paradox is resolved by keeping track of the motion of any particular spin due to a family of skyrmion trajectories. Assume the skyrmion first passes our chosen spin on its right, but then the trajectories gradually sweep across the spin so that the skyrmion eventually passes the spin on its left. We see that the corresponding family of spin paths on $S^{2}$ start with a small loop near the south pole, then, as the skyrmion passes closer to the spin, the loop in $S^{2}$ grows in area, circling the equator of the sphere when the center of the skyrmion goes exactly through the spin. As the skyrmion passes further to the left the loop begins to shrink towards the south pole again, but the area enclosed by the loop continues to grow, reaching $4 \pi$, or the entire sphere, when the skyrmion passes far to the left. Thus, although the spins far to the left of the skyrmion's trajectory hardly move, they must be counted as contributing $4 \pi$ to $\oint \dot{\mathbf{n}} \cdot \mathbf{A} d t$. 


\section{Quantum Hall effect}

We now wish to examine the dynamics of the skyrmions in the quantum Hall effect. We will describe this system by means of the Zhang-Hansson-Kivelson (ZHK) model [9], as modified by Kane and Lee [10] to take into account the effects of spin. This is one of the models that was used by Sondhi et al. [1].

We will take as lagrangian for this system

$$
\begin{gathered}
L=i \phi^{\dagger}\left(\partial_{0}-i\left(a_{0}+e A_{0}\right)\right) \phi-\frac{1}{2 m^{*}}\left|\left(\partial_{i}-i\left(a_{i}+e A_{i}\right)\right) \phi\right|^{2} \\
-\frac{\lambda}{2}\left(|\phi|^{2}-\rho_{0}\right)^{2}+\frac{1}{4 \Theta} \epsilon^{\mu \nu \sigma} a_{\mu} \partial_{\nu} a_{\sigma}
\end{gathered}
$$

Here $\phi=\left(\phi_{1}, \phi_{2}\right)$ is a two-component complex scalar field. The quantity $\Theta$ is the statistics parameter which must take one of the values $\Theta=(2 n+1) \pi$ in order that the boson field $\phi$ represents a fermion. $A_{\mu}$ is the external electromagnetic field and $m^{*}$ is the effective mass of the electron. Repeated roman indices imply sums over the spatial directions $i=1,2$, while repeated greek indices imply sums over both space and time directions $\mu=0,1,2$.

We should also include Coulomb and Zeeman interactions. These are essential for determining the energy and size of the skyrmions, but they are not important ingredients in the topological effects we are studying here. We will therefore omit them from our expressions so as not to unduly clutter our formulae.

In order to find a solution with uniform density $\rho \equiv\left(\left|\phi_{1}\right|^{2}+\left|\phi_{2}\right|^{2}\right)=\rho_{0}$ at filling fraction $\nu=1 /|2 n+1|$ we must adjust the magnetic field $B_{z}=\partial_{1} A_{2}-\partial_{2} A_{1}$ so that $e B_{z}=2 \Theta \rho_{0}$, with the sign of $\Theta=(2 n+1) \pi$ chosen so as to ensure $\rho_{0}>0$. At this magic value the Chern-Simons field cancels the effects of the external magnetic field on $\phi$. We assume that this adjustment has been made, and that the solution selected by the Zeeman term has $\phi_{1}=\sqrt{\rho_{0}}, \phi_{2}=0$. We now examine the dynamics of the fluctuations about this uniform solution under the conditions that the density varies sufficiently slowly that derivatives of $\rho$ can be ignored. (Including the derivatives of $\rho$ merely adds a "quantum pressure" of the kind familiar from the Gross-Pitaevski model of a quantum fluid.) We therefore replace (3.1) by a lagrangian reminiscent of the $C P^{1}$ version of the non-linear $\sigma$-model $[10]$

$$
\begin{gathered}
L=i \rho \mathbf{z}^{\dagger}\left(\partial_{0}-i\left(a_{0}+e A_{0}\right)\right) \mathbf{z}-\frac{\rho}{2 m^{*}}\left|\left(\partial_{i}-i\left(a_{i}+e A_{i}\right)\right) \mathbf{z}\right|^{2} \\
-\frac{\lambda}{2}\left(\rho-\rho_{0}\right)^{2}+\frac{1}{4 \Theta} \epsilon^{\mu \nu \sigma} a_{\mu} \partial_{\nu} a_{\sigma} .
\end{gathered}
$$

Here $\mathbf{z}$ is a two component complex field with the constraint $\mathbf{z}^{\dagger} \mathbf{z}=\left|z_{1}\right|^{2}+\left|z_{2}\right|^{2}=1$. We can now partially decouple the spin dynamics from the charge transport by using the identity

$$
\frac{\rho}{2 m^{*}}\left|\left(\partial_{i}-i\left(a_{i}+e A_{i}\right)\right) \mathbf{z}\right|^{2}=\frac{\rho}{8 m^{*}}(\nabla \mathbf{n})^{2}+\frac{m^{*}}{2 \rho} \mathbf{J}^{2},
$$


where $\mathbf{J} \equiv\left(J^{1}, J^{2}\right)$ with

$$
J^{i}=\frac{\rho}{m^{*} i}\left(\mathbf{z}^{\dagger} \partial_{i} \mathbf{z}-i\left(a_{i}+e A_{i}\right)\right)
$$

is the number current, $n^{a}=\mathbf{z}^{\dagger} \sigma^{a} \mathbf{z}$ is the local spin direction, and $\nabla \mathbf{n} \equiv\left(\partial_{1} \mathbf{n}, \partial_{2} \mathbf{n}\right)$.

Our lagrangian now looks like

$$
L=i \rho\left(\mathbf{z}^{\dagger} \partial_{0} \mathbf{z}-i\left(a_{0}+e A_{0}\right)\right)-\frac{\rho}{8 m^{*}}(\nabla \mathbf{n})^{2}-\frac{m^{*}}{2 \rho} \mathbf{J}^{2}+\ldots
$$

where the dots represent the potential and Chern-Simons terms that are temporarily uninteresting.

We next demonstrate that (3.5) represents the action for a ferromagnet of the kind considered in section 2. We already have a spin stiffness term with $K=\rho / 4 m^{*}$, and we will now show that the time derivatives of $z$ provide the $\dot{\mathbf{n}} \cdot \mathbf{A}(n)$ term.

The expression appearing as part of the currents and in the kinetic term, $K_{\mu} \equiv \mathbf{z}^{\dagger} \partial_{\mu} \mathbf{z}$ is essentially the Berry connection on $S^{2}$. It is no surprise, then, that $K_{\mu}$ satisfies

$$
\partial_{\mu} K_{\nu}-\partial_{\nu} K_{\mu}=\frac{i}{2} \mathbf{n} \cdot\left(\partial_{\mu} \mathbf{n} \wedge \partial_{\nu} \mathbf{n}\right) .
$$

We can use this equation to find the variation of $K_{\mu}$ under a variation of the direction $\mathbf{n}$. Replacing one of the partial derivatives by $\delta$ gives us

$$
\delta K_{\mu}=\frac{i}{2} \mathbf{n} \cdot\left(\delta \mathbf{n} \wedge \partial_{\mu} \mathbf{n}\right)+\partial_{\mu} \Lambda
$$

where $\Lambda=\mathbf{z}^{\dagger} \delta \mathbf{z}$. To be rather more concrete we could write $\mathbf{z}=U \mathbf{z}_{0}$ where $\mathbf{z}_{0}=\left(\begin{array}{l}1 \\ 0\end{array}\right)$ and $U \in S U(2)$. We make a variation in $U$ so that $\delta U U^{-1}=i \delta \mathbf{w} \cdot \sigma / 2$ and find that

$$
\begin{aligned}
\delta \mathbf{n} & =\mathbf{n} \wedge \delta \mathbf{w} \\
\delta K_{\mu} & =-\frac{i}{2} \delta \mathbf{w} \cdot \partial_{\mu} \mathbf{n}+\frac{i}{2} \partial_{\mu}(\mathbf{n} \cdot \delta \mathbf{w}) .
\end{aligned}
$$

However we make the variation, the second term in $\delta K_{\mu}$ is a pure gauge transformation, and so has no effect on the lagrangian provided the number conservation equation $\dot{\rho}+\nabla \cdot \mathbf{J}$ is satisfied. Substituting the variation from (3.8) into (3.5) (and again ignoring gradients of $\rho$ ) gives us

$$
\frac{\rho}{2}\left(\partial_{t}+\mathbf{v} \cdot \nabla\right) \mathbf{n}=\frac{\rho}{4 m^{*}} \mathbf{n} \wedge\left(\nabla^{2} \mathbf{n}\right)
$$

where $\mathbf{v}=\mathbf{J} / \rho$ is the local electron-fluid velocity.

We see that (3.9) is the Landau-Lifshitz equation for a density $\rho$ of spin- $\frac{1}{2}$ particles - except that the time derivative has been replaced by a convective derivative. (The $(\mathbf{v} \cdot \nabla) \mathbf{n}$ term comes from the variation 
of $\mathbf{J}^{2}$ in (3.5).) This modification is to be expected because the galilean invariance implicit in (3.1) requires the spin waves to be carried along with the local flow.

Provided the electron fluid remains stationary, the spins in the quantum Hall system obey exactly the same equation as in a conventional ferromagnet. The solitons, although they are electrically charged, must therefore experience only the same Magnus force that they feel in the neutral ferromagnet. Now the skyrmion does carry its charge along with it so a small velocity field is induced by the skyrmion motion - but this does not affect our conclusions as we will now show by making a partial duality transformation.

We first promote the current, $\mathbf{J}$, to the status of an independent dynamical variable by making a Hubbard-Stratanovich transformation. The lagrangian becomes

$$
\begin{aligned}
L=i \rho\left(\mathbf{z}^{\dagger} \partial_{0} \mathbf{z}\right. & \left.-i\left(a_{0}+e A_{0}\right)\right)+i\left(\mathbf{z}^{\dagger} \partial_{i} \mathbf{z}-i\left(a_{i}+e A_{i}\right)\right) J^{i}+\frac{m^{*}}{2 \rho} \mathbf{J}^{2}-\frac{\lambda}{2}\left(\rho-\rho_{0}\right)^{2} \\
+ & \frac{1}{4 \theta} \epsilon^{\mu \nu \sigma} a_{\mu} \partial_{\nu} a_{\sigma}-\frac{\rho}{8 m^{*}}(\nabla \mathbf{n})^{2} .
\end{aligned}
$$

Integrating over the $U(1)$ phase degree of freedom in $\mathbf{z}$ enforces the current conservation law as a constraint, so we can write the current/density three-vector $\left(\rho \equiv J^{0}, J^{1}, J^{2}\right)$ as the curl of a three-dimensional vector field. We set $J_{\{0\}}^{\mu} \equiv\left(\rho_{0}, 0,0\right)$ equal to $\epsilon^{\mu \nu \sigma} \partial_{\nu} \mathcal{A}_{\sigma}^{\{0\}}$ and

$$
J^{\mu}-J_{\{0\}}^{\mu}=\epsilon^{\mu \nu \sigma} \partial_{\nu} \mathcal{A}_{\sigma} .
$$

At this point we also integrate out the Chern-Simons field $a_{\mu}$. After some further integration by parts and use of the relation $2 \Theta \rho_{0}=e B_{z}$ we find

$$
L=2 \pi \mathcal{J}^{\mu}\left(\mathcal{A}_{\mu}+\mathcal{A}_{\mu}^{\{0\}}\right)-\Theta J^{\mu} \mathcal{A}_{\mu}+\frac{m^{*}}{2 \rho} \mathbf{J}^{2}-\frac{\lambda}{2}\left(J^{0}-J_{\{0\}}^{0}\right)^{2}-\frac{\rho}{8 m^{*}}(\nabla \mathbf{n})^{2},
$$

where

$$
\mathcal{J}^{\mu}=\frac{1}{2 \pi i} \epsilon^{\mu \nu \sigma} \partial_{\mu} \bar{z}_{\alpha} \partial_{\nu} z_{\alpha}
$$

is the skyrmion number current.

We can make the physics content of (3.12) clearer by first linearizing by setting $\rho=\rho_{0}$ in the kinetic energy term. Then, for cosmetic reasons, we adjust the units of length and time so that $c \equiv \sqrt{\lambda \rho_{0} / m^{*}}$, the velocity of density waves in the absence of the magnetic field, becomes unity, and define the field strength tensor $\mathcal{F}_{\mu \nu}=\partial_{\mu} \mathcal{A}_{\nu}-\partial_{\nu} \mathcal{A}_{\mu}$ to be the dual of the electron number current.

With these changes we have

$$
L=2 \pi \mathcal{J}^{\mu}\left(\mathcal{A}_{\mu}+\mathcal{A}_{\mu}^{\{0\}}\right)-\frac{1}{2} \Theta \epsilon^{\mu \nu \sigma} \mathcal{A}_{\mu} \mathcal{F}_{\mu \nu}-\frac{\lambda}{4} \mathcal{F}_{\mu \nu} \mathcal{F}^{\mu \nu}-\frac{1}{8 \lambda}(\nabla \mathbf{n})^{2} .
$$


We see that the skyrmion number current acts a source for a topologically massive gauge field $\mathcal{A}_{\mu}$ [11], and also sees the background field $\mathcal{A}^{\{0\}}$. The curl of $\mathcal{A}^{\{0\}}$ is equal to both $\rho_{0}$ and $e B_{z} / 2 \Theta$, so the interaction with the background field provides a phase for each skyrmion world line that can be interpreted as producing either the Magnus force on the skyrmion, or, provided that the skyrmion number current and the electron number current associated with the skyrmion world line are proportional with proportionality factor $\Theta / \pi$, the Lorentz force. The interaction with the topologically massive gauge field provides a phase factor that depends on all skyrmions present and gives the skyrmion Fermi (for $\nu=1$ ), or anyon (for $\nu=\frac{1}{|2 n+1|}$ ) statistics. The topologically massive gauge field also has its own degrees of freedom. These are the gapped magneto-phonons or magneto-plasmon density fluctuation modes.

If we momentarily freeze out the density fluctuation modes by ignoring the $\mathcal{F}_{\mu \nu} \mathcal{F}^{\mu \nu}$ term in (3.14), the equation of motion from varying $\mathcal{A}_{\mu}$ is

$$
2 \pi \mathcal{J}^{\mu}=\Theta \epsilon^{\mu \nu \sigma} \mathcal{F}_{\nu \sigma}=2 \Theta J^{\mu}
$$

This shows that, up to the expected factor that reflects the skyrmion possessing total charge $\frac{1}{2 n+1}$, the topological current density coincides with the electron current density. If we now reintroduce the $\mathcal{F}_{\mu \nu} \mathcal{F}^{\mu \nu}$ term the two current densities no longer exactly coincide, but, because the extra term that appears in the equation of motion is a pure divergence, the total currents flowing along a skyrmion world line are the same. This confirms that the Magnus force and Lorentz force on the skyrmion are indeed equal, and that they are merely different physical interpretations of the same phase factor.

\section{Discussion}

The same disappearing-force phenomenon occurs for the vortex-like charged quasiparticles in the original ZHK model. These quasiparticles also look as if they should experience a Magnus force, both because of the phase winding in the order parameter and because of there is circulation around the vortex. They should also be acted on by a Lorentz force because of their charge. Once again only one force survives [12]. In this case however the effect is not so startling. The circulation vanishes at large distance from the quasiparticles and, anyway, they are very much children of the quantum Hall phase and one is less likely to grant them attributes outside it.

Skyrmions on the other hand have an independent existence in planar ferromagnets. They experience a Magnus force when neutral and so would naively be expected to experience an additional Lorentz force when given a charge. It requires an intricate conspiracy for the two forces to become one. Such intricacies are not unknown in the ferromagnets. Identifying the force on a soliton is equivalent to identifying the 
momentum that the force is changing. For the Landau-Lifshitz system this is non-trivial. In the continuum approximation to a ferromagnet with spins in fixed locations the momentum operator $\hat{P}$ itself is not welldefined. Only the translation operator $T_{a}=\exp \{i a \hat{P}\}$ makes sense, and then only for translations through a distance $a$ that takes one past an integer number of $J=\frac{1}{2}$ spins [13]. When the spins are attached to mobile electrons, as they are in the Hall effect, the system is manifestly translation invariant and must therefore have a well-defined momentum. There is, however, no gauge invariant separation between the momentum residing in the collective orientational order of the spins and the motion of the electrons [14].

In [14] Volovik pointed out that, once a gauge tranformation has been applied to align the local spin quantization frame along $\mathbf{n}$, the electrons respond to the winding-number density of $\mathbf{n}$ as if it were a magnetic flux. This observation provides a simple picture of what is happening here. A moving flux produces an electric field and in Volovik's one-dimensional example the passage of a skyrmion accelerates the electrons, producing a spectral flow that effectively transfers a single electron from one side of the Fermi surface to the other. His electrons have thus gained momentum $2 k_{f}=2 \pi \rho$.

A two-dimensional version of this phenomenon is the physical origin of the Magnus force. For a ferromagnet composed of mobile electrons with an ungapped fermi-surface the electrons gain both energy and momentum from the moving skyrmion. A force must be applied to the skyrmion to provide this momentum and energy. When the electron gas is in a quantum Hall phase, however, no change in occupation number through spectral flow is possible because there is a gap in the spectrum. The skyrmion is therefore no longer able to excite the system — but the moving "flux" is still attempting to transfer mechanical momentum to the electron fluid. The electrons are unable to accelerate in response because they are locked in place by the external magnetic field. The mechanical momentum generated by the changing "flux" must therefore be transferred to the magnet that creates this field. Now exerting a force on the magnet requires the electrons to produce a real magnetic field. Fortunately the Hall effect itself requires the geometric "flux" to accumulate extra charge in its vicinity and the electrons are able to generate the necessary field from the current created by dragging this charge along with the skyrmion. Since magnets obey Newton's third law, the magnet then produces an equal and opposite force on the skyrmion.

By this sequence of maneuvers the Hall effect electrons have transformed the original Magnus force into a Lorentz force.

Note added: Since finishing this manuscript I became aware that the Magnus force is briefly discussed in a recent paper by Read and Sachdev [15]. 


\section{Acknowledgements}

The question addressed in this paper arose in a discussion with Amir Caldeira. Most of the work was carried out at the Erwin Schrödinger Institute in Vienna and I would like to the thank the staff and members of the ESI for their hospitality. I must also thank Eduardo Fradkin and Frank Gaitan for discussions about both the physics and the text. This work was supported by the National Science Foundation under grant DMR94-24511.

\section{References}

[1] S. L. Sondhi, A. Karlhede, S. A. Kivelson, E. H. Rezayi, Phys. Rev. B47 (1993) 16419.

[2] S. E. Barrett, G. Dabbagh, L. N. Pfeiffer, W. W. West, R. Tycko, Phys. Rev. Lett. 74 (1995) 5122; R. Tycko, S. E. Barrett, G. Dabbagh, L. N. Pfeiffer, K. W. West, Science, 268 (1995) 1460.

[3] J. R. Klauder, Phys. Rev. D 19 (1979) 2349.

[4] For a recent account see: E. A. Kochetov, J. Math. Phys. 36 (1995) 4667.

[5] M. Stone, Nucl. Phys. B314 (1989) 557

[6] A. A. Kirillov, Elements of the Theory of Representations, (Springer-Verlag 1976).

[7] L. D. Landau, E. M. Lifshitz, Phys. Z. Sowjet, 8 (1935) 3470; W. Döring, Z. Phys 124 (1947) 501; C. Herring, Ch. Kittel. Phys. Rev., 81 (1951) 869.

[8] For a review see R. Rajaraman Solitons and Instantons, (North Holland, Amsterdam 1982 )

[9] S.-C. Zhang, T. H. Hansson, S. A. Kivelson, Phys. Rev. Lett., 62 (1989) 82.

[10] D. H. Lee, C. L. Kane, Phys. Rev. Lett. 64 (1990) 1313.

[11] J. Schonfield, Nucl. Phys. B 185 (1981) 157; S. Deser, R. Jackiw, S. Templeton, Annals of Physics (NY) 140(1982) 372

[12] M. Stone, Phys. Rev. B42 (1990) 212.

[13] F. D. M. Haldane, Phys. Rev. Lett. 57 (1986) 1488.

[14] G. E. Volovik, J. Phys C20 (1987) L83 
[15] N. Read, S. Sachdev, Phys. Rev. Lett. 75 (1995) 3509. 